\title{
Blind Video Watermarking based on DWT-SHUR and Optimized Firefly Algorithm
}

\author{
Akanksha Dixit \\ M.Tech. Scholar \\ Sachdeva Institute of \\ Technology, \\ Mathura Uttar Pradesh, \\ India
}

\author{
Pankaj Sharma, PhD \\ Sachdeva Institute of \\ Technology, \\ Mathura Uttar Pradesh, \\ India
}

\author{
Vyom Kulshreshtha \\ Sachdeva Institute of \\ Technology, \\ Mathura Uttar Pradesh, \\ India
}

\begin{abstract}
Illegitimate duplication, piracy, circulation and amendment of digitized information are well thought-out infringements aligned with intellectual property rights. Thus digital watermarking came into existence due to the evolving obligation of copyright protection.

We have presented a blind digital video watermarking technique based on the Discrete Wavelet transform (DWT), Firefly algorithm and the real Schur Decomposition. The scheme is using optimized firefly algorithm to trace the best DWT blocks with large texture value for inserting watermark and to minimize the error rate. The scheme starts with applying two-level DWT to the video scene. It finds the suitable place to embed watermark using Firefly algorithm and then apply Schur decomposition. Schur uses Singular Value Decomposition to embed the binary watermark bits in the resultant block upper triangular matrix. The proposed technique shows high efficiency since Schur decomposition requires fewer computations compared to other transforms and robustness due to optimized firefly algorithm. It provides better results in terms of imperceptibility and normalized correlation.
\end{abstract}

\section{General Terms}

Digital Watermarking, Authentication, Security, Algorithms et. al.

\section{Keywords}

Discrete Wavelet Transform (DWT), Firefly Algorithm, SCHUR Decomposition, Normalized correlation

\section{INTRODUCTION}

Digital watermarking is a technique that inserts information, such as authentication and ownership information into digital data to avert on or after illicit copying.

Effectiveness of watermarking techniques is measured in terms of both imperceptivity and robustness of the algorithm.

Imperceptibility indicates the watermark perceptual transparency [1], while robustness refers to the ability of extracting the embedded watermark from the received media even after an intentional or unintentional attack such as degradation, additive noise, rotation, cropping, or filtering[1] [2]. Two additional considerable issues of the watermarking techniques are data payload and normalized correlation, Data payload, also referred as capacity, includes methods that make it possible to embed the large information. Normalized correlation compares the similarities between the original and extracted watermarks [3][11]

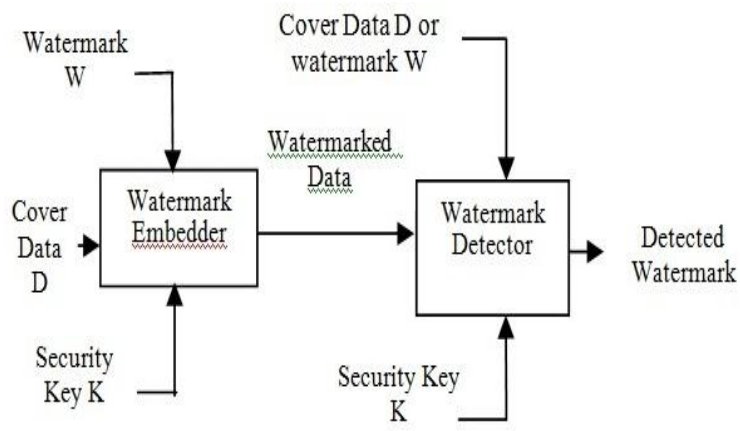

Fig 1: A typical watermarking system

\section{VIDEO FRAMES AND VIDEO} WATERMARKING

In any digital watermarking technique, the position or place where the watermark is embedded, is termed as host. The host can be any digital media like image, audio or video. A video sequence constructs from multiple frames where each frame can be considered as an image. So image watermarking can be extended to video watermarking. The watermark in the proposed technique is embedded in the frames of a video clip.

\subsection{Discrete Wavelet Transform}

Discrete Wavelet Transform is the multi resolution description of an image, where the decoding can be processed sequentially from a low resolution to the higher resolution [4]. The discrete wavelet transform is based on wavelets, which are basically small waves, of varying frequency and limited duration. Such wavelet transform provides frequency as well as spatial description of an image [5]. The Discrete Wavelet Transform DWT divides the signal into frequency ranges or parts as high and low. The high frequency part contains information about the edge components, while the low frequency part is split again into high and low frequency parts. The discrete wavelet transform (DWT) splits the input image hierarchically to four frequency levels. One level is low (LL) and the other three levels are high: HL, LH, and HH as shown in Figure 2.

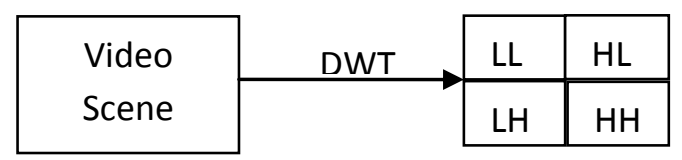

Fig 2: 1-level DWT sub-bands of Frame.

An image is a two dimensional function that can be decomposed by DWT in multiple levels. In the proposed technique we applied 2 level DWT on the image in the first 
level, followed by the High level (HL) band in the second level. Each sub-band represents a matrix of DWT coefficients at a particular resolution. Figure 3 shows the sub-bands produced by the 2-level DWT decomposition.

\begin{tabular}{|c|c|c|}
\hline \multirow{2}{*}{ LL1 } & LL2 & HL2 \\
\cline { 2 - 3 } & LH2 & HH2 \\
\hline \multirow{2}{*}{ LH1 } & \multicolumn{2}{|c|}{ HH1 } \\
& \multicolumn{2}{|c|}{} \\
\hline
\end{tabular}

Fig 3: 2-level DWT sub-bands of Frame.

\subsection{Schur Decomposition}

The Schur decomposition also referred as Schur triangulation is an important mathematical tool in the field of linear algebra. The method, named after Issai Schur, is used in metrics analysis. SCHUR decomposition represents a major intermediate step in SVD (singular value decomposition) [7]; and it is an efficient mathematical tool as for an $\mathrm{n} * \mathrm{n}$ matrix it requires only about $\mathrm{n}^{3}$ flops as compared to SVD decomposition that requires $11 \mathrm{n}^{3}$ flops. So the computational effort in Schur decompostion is just one third of as in SVD decomposition [8]. Schur decomposition has two version: complex Schur and real Schur, [9]. The Real Schur decomposition version of a real square matrix A results in two matrices: $\mathrm{U}$ and $\mathrm{S}$ as shown in Equation (1):

$\operatorname{Schur}(\mathrm{A})=\left(\mathrm{U} \times \mathrm{S} \times \mathrm{U}^{\mathrm{T}}\right)$

where $S$ refers to the block upper triangular matrix called the real Schur form and $\mathrm{U}$ indicates a unitary orthogonal matrix. UT represents the conjugate transpose of U. The following example illustrates the Schur decomposition applied on a $3 \times$ 3 matrix A.

$A=\begin{array}{lll}160 & 160 & 159 \\ 160 & 159 & 159 \\ 161 & 160 & 158\end{array}$

Applying real Schur decomposition on matrix A will produce both $\mathrm{U}$ and $\mathrm{S}$ matrices as follows:

$$
\mathrm{U}=\begin{array}{ccc}
0.5778 & 0.4077 & 0.7071 \\
0.5765 & 0.8171 & 0.0000 \\
0.5778 & 0.4077 & 0.7071 \\
478.6664 & 0.7071 & 2.0418 \\
\mathrm{~S}= & 0.6664 & 0.5753 \\
0 & 0 & 1.0000
\end{array}
$$

Apparently, Matrix $\mathrm{S}$ is a triangular matrix; and it is also similar to A by definition of matrices similarities [7]. Accordingly, matrix $\mathrm{S}$ has the same multi set of Eigen values in A. Eigen values set is contained in the main diagonal of the $\mathrm{S}$ matrix because $\mathrm{S}$ is triangular. Since Eigen values in Schur decomposition are highly stable, the diagonal in $\mathrm{S}$ matrix was selected in our technique for embedding watermark [9].

\subsection{Firefly Algorithm}

The projected method is resting on firefly algorithm which impersonates the behavior of fireflies. In the humid and moderate area during summer Fireflies flash light in sky. The intensity of the flashes created by means of the fireflies is the source in favor of this algorithm. They articulate each other with the assistance of intensity of flashes and fireflies attempt to progress in the path of the fireflies encompassing high intensity of flashes. With the distance the light intensity diverge from the other fireflies and a petite intensity is missing in middle. Light intensity is computed in this algorithm in the existence of a few variables in aid of intensity mislaid and distance among fireflies. The algorithm generate optimal outcome with a reduction of computational time and is enough random in nature.

The Firefly Algorithm is a latest nature encouraged procedure $[10,11]$ that has commonly been used for solving NP tough optimization troubles. Xin-She Yang projected this Algorithm. Firefly algorithm is just elucidated by the consequent three idealized principles:

a) Each and every Firefly is unisex with the intention that one Firefly will be concerned to other Fireflies despite of their sex.

b) Attractiveness is relative to their intensity, as a result for any two flashing Fireflies, the less bright Firefly will progress in the track of the brighter Firefly. The attractiveness is comparative to the intensity and they mutually turn down as their distance rises. Firefly will travel randomly, if there is no particular Firefly which is brighter than the existing one.

c) The intensity of a Firefly is resolute by the background of the objective function.

\section{THE PROPOSED OPTIMIZED DWT- SCHUR TECHNIQUE}

In this paper a blind robust digital video watermarking technique is proposed. The proposed technique applies both DWT and Schur transforms on the digital video. The binary watermark is embedded in the video frames. The Firefly algorithm is used to detect the suitable position for embedding watermark in the frame. The proposed DWT-Schur watermarking algorithm has two procedures; one is to embed the watermark into the original video frame, while the other to extract the watermark from the received watermarked version of the frame.

\subsection{Firefly Algorithm flow}

In the proposed method, the FA is used to trace the best DWT blocks with large texture value for inserting watermark and to minimize the error rate. The maximum optimization algorithm of the Firefly is depicted below. Earliest initial population of candidate solutions is created. Later, it estimate the light intensity for the entire Fireflies and locate the attractive Firefly (finest candidate) surrounded by the population. Followed by, analyze the attractiveness and distance for all Fireflies to progress all Fireflies towards the attractive Firefly in the explore region. As a final point, the attractive Firefly progress randomly in the explore region. This sequence is continual in anticipation till a termination criterion is met i.e., the utmost numeral of generations is attained.

Delineate Objective function $\mathrm{f}(\mathrm{x}), \mathrm{x}=(\mathrm{x} 1, \ldots, \mathrm{xd})^{\mathrm{T}}$

Build initial population of fireflies $\mathrm{x}_{\mathrm{k}}(\mathrm{k}=1,2, \ldots, \mathrm{p})$

Delineate $\alpha, \beta$, highest generation numeral $(\mathrm{HN})$ and $\gamma$

Output: The preeminent result $\mathrm{x}_{\mathrm{k}} \max$ with the leading objective function value

Begin

while $(\mathrm{t}<\mathrm{HN})$

for $\mathrm{u}=1: \mathrm{p}$ all $\mathrm{p}$ fireflies

for $\mathrm{v}=1: \mathrm{p}$ all $\mathrm{p}$ fireflies 
if $f(x u)<f(x v)$

calculate attractive fireflies by eq. (2)

calculate the distance between fireflies $\mathrm{u}$ and $\mathrm{v}$ by eq. (3)

move fireflies to the best solution by eq. (4)

else

move fireflies to the best solution randomly by eq. (5)

assess novel solutions and keep posted objective function

end if

end $\mathrm{v}$

end $\mathrm{u}$

end while

grade the fireflies and locate the existing global best $\mathrm{x}_{\mathrm{u}} \max$

End

The process commence on or after an initial population of randomly created individuals. The eminence of each individual is calculated via Eq. (14) and the preeminent solution between them is chosen. In Firefly Algorithm, the type of attractiveness function of a firefly is represented by the succeeding:

$\beta(d)=\beta_{0} e^{-\gamma D m}$

where, $\mathrm{d}=$ The distance among any two Fireflies

$\beta_{0}=$ The early attractiveness at $d=0$

$\gamma=$ light absorption coefficient which direct the decline of the light intensity

The distance connecting any two Fireflies $u$ and v, at positions and correspondingly, can be termed as a Cartesian or Euclidean distance as go after:

$\mathrm{d}_{\mathrm{pq}}=\mathrm{x}_{\mathrm{p}}-\mathrm{x}_{\mathrm{q}}=\sqrt{\sum_{k-1}^{p}(x(p, k)-x(q, k))^{2}}$

The movement of a Firefly u which is attracted by another brighter Firefly v correspond to the subsequent equation:

$\mathrm{x}_{\mathrm{u}}=\mathrm{x}_{\mathrm{u}}+\beta_{0} \mathrm{e}^{-\gamma \mathrm{dpq}}\left(\mathrm{x}_{\mathrm{q}}-\mathrm{x}_{\mathrm{p}}\right)+\alpha\left(\right.$ rand $\left.-\frac{1}{2}\right)$

If there are no Fireflies brighter than a particular Firefly u with maximum objective value then $u$ will move randomly according to the Eq. (17).

where $\mathrm{x}_{\mathrm{umax}}=\mathrm{x}_{\mathrm{umax}}+\alpha\left(\right.$ rand $\left.-\frac{1}{2}\right) \mathrm{u}_{\max }$ (5)

In Eq. (4), the initial term is the recent location of a Firefly, the second term is utilized in view of the attractiveness of a Firefly, in the path of the intensity of light by nearby Fireflies and when it lacks the brighter ones, the third term is exploited for the random progress of a Firefly (random part). The coefficient $\alpha$ is a randomization factor resolute by the problem of curiosity, despite the fact that rand is a random number generator time after time disseminated in the space $(0,1)$. In Eq. (5), the progress of the preeminent candidate is finished randomly.

\subsection{Watermarking Embedding Procedure}

The proposed Watermarking Algorithm based upon DWT, Schur and Firefly algorithm begins with splitting the video into video scenes, followed by embedding the selected binary watermark into the video frames. The frame to be watermarked is selected by Firefly algorithm.

\section{Input Video}

1. Input the video clip V.

2. Divide the video clip into video scenes Vsi

3. Select Apply DWT on the frames of each Vsi using DWT, Firefly algorithm, and Schur as described in steps $4-12$.

4. Convert the color matrix format from RGB to YUV for every video frame $\mathrm{F}$.

\section{Apply 2-level DWT}

5. For the $\mathrm{Y}$ (luminance) matrix in each frame F, compute the 2-level DWT. This operation generates seven DWT sub- bands [LL1, LL2, HL2, LH2, HH2, LH1, HH1].

\section{Apply SCHUR}

6. Apply the Schur operator on the HL2 sub-band. The Schur operator decomposes the sub-band's coefficient matrix into two independent matrices:

$$
\mathrm{HL} 2=\mathrm{U}_{\mathrm{HL} 2} \mathrm{~S}_{\mathrm{HL} 2}
$$

\section{Embedding}

7. Apply Fireflies algorithm to find the location of DWT block with large texture for embedding the watermark. This secures the transparency.

8. Rescale the watermark image so that the size of the watermark will match the size of the HL2 sub-band which will be used for embedding.

9. Embed the binary bits of watermark WVsi into the diagonal matrix of SHL2 by substituting the watermark bit Wi with the eighth LSB (Least significant Bit) bit of SHL2 (i, i):

$$
\operatorname{LSB}\left(\mathrm{S}_{\mathrm{HL} 2}(\mathrm{i}, \mathrm{i})\right)=\mathrm{W}_{\mathrm{VSi}}
$$

\section{Video Reconstruction}

10. Apply the inverse Schur operator on the modified $S^{\prime}$ HL2 matrix to get a modified coefficient matrix HL2'. The inverse Schur operation is as follows:

UHL2 $\times$ SHL $2 \times$ UTHL2)

11. Apply the inverse DWT on the modified coefficient matrix HL2'. This operation produces the final watermarked Video frame F'.

12. Convert the video frames $f^{\prime}$ from YUV to RGB color matrix.

13. Reconstruct frames into the final watermarked Video scene F'.

14. Reconstruct watermarked scenes to get the final watermarked video clip V'

The embedding procedure described previously is depicted in the block diagram shown in Figure 12. 


\subsection{Watermark Extraction Procedure}

The proposed DWT-Schur algorithm is completely blind as the Watermark extraction procedure does not require the original. Consequently, we can extract the watermark image directly from the LSBs in the watermarked video frames. The watermarking extraction procedure is described in details below.

\subsubsection{Extraction procedure steps}

\section{Input Video}

1. Input the watermarked Video clip $\mathrm{V}^{\prime}$.

2. Split the watermarked Video clip $\mathrm{V}^{\prime}$ into watermarked scenes

3. Vsi'.

4. Convert the video frame $\mathrm{F}^{\prime}$ from RGB color matrix to YUV.

5. Perform the DWT and Schur decomposition on the watermarked frames of each watermarked video scene as described in steps $5-7$.

\section{Apply 2-level DWT}

5. The frame selected with large texture value denoted as F' by using Firefly optimization where watermark is embedded. Compute the 2-level DWT for the frame $\mathrm{F}^{\prime}$. This will produce the seven sub-bands as: [wLL1, wLL2, wHL2, wLH2, wHH2, wLH1, wHH1].

\section{Apply SCHUR}

6. Apply the Schur operator on the wHL2 sub-band. The Schur operator decomposes the sub-band's coefficient matrix into two independent matrices:

$\mathrm{W}_{\mathrm{HL} 2}=\mathrm{U}_{\mathrm{WHL} 2} \mathrm{~S}_{\mathrm{WHL} 2}$

.................(9)

\section{Extraction}

7. Extract the embedded watermark from the diagonal matrix wHL2 $\mathrm{S}$ as follows:

$\mathrm{W}_{\mathrm{VSi}}(\mathrm{i})=\mathrm{LSB}\left(\mathrm{S}_{\mathrm{HL} 2}(\mathrm{i}, \mathrm{i})\right)$

\section{Video Reconstruction}

8. Construct the image watermark WVsi by cascading all watermark bits extracted from all frames.

9. Repeat the same procedure for all video scenes.

The above proposed watermarking extraction procedure is illustrated in the block diagram shown in Figure 4.

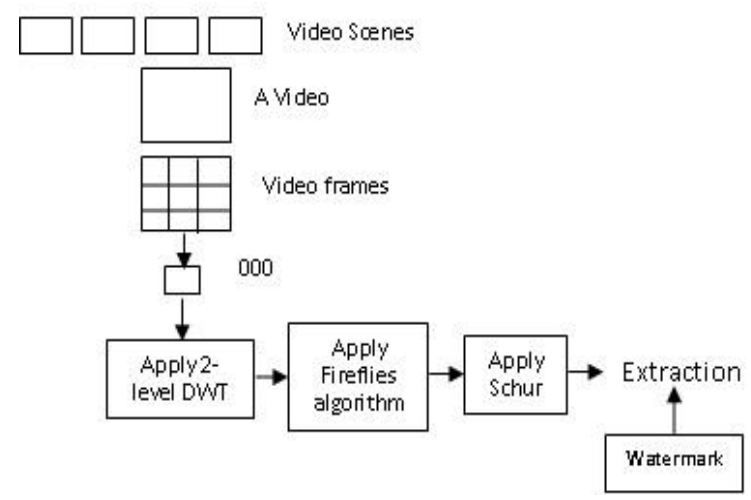

Fig 4: The watermark extraction procedure.

\section{EXPERIMENTAL RESULTS AND ANALYSIS}

\subsection{Imperceptibility test}

To evaluate the imperceptibility of the projected method, a succession of tests has been accomplished. The watermark image taken for inserting into the videos is depicted in Fig.5. Original videos taken for testing and the corresponding Watermarked videos obtained after inserting the watermark are exposed in Fig.6. In the experiments, no visible artifacts can be empirical in all of the test video sequences. In addition to subjective inspection, PSNR (Peak Signal to Noise Ratio) is typically in use to estimate the perceptual quality. Fig.7. exemplifies the Y-PSNR (luma PSNR) discrepancy provoked by inserting the watermark even after different types of attacks for the three different videos. We examine that the watermarked video is not discernible as of the original video. Fig.7. depicts the PSNR values for distinct videos and we view that it is nearly not viable to distinguish the degradation in video quality caused by inserting the watermark even after variety of attacks

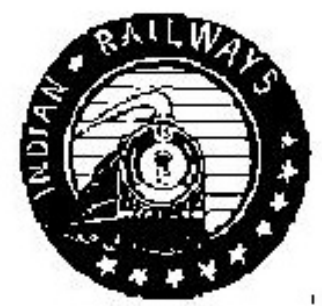

Fig 5: Watermark Image 


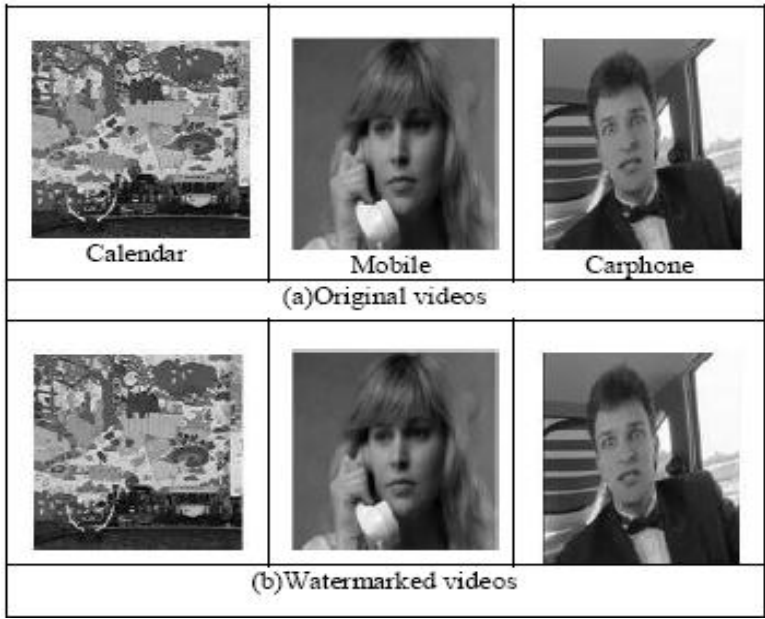

Fig 6 (a): Original videos and (b)Watermarked videos of Calendar, Mobile and Carphone.

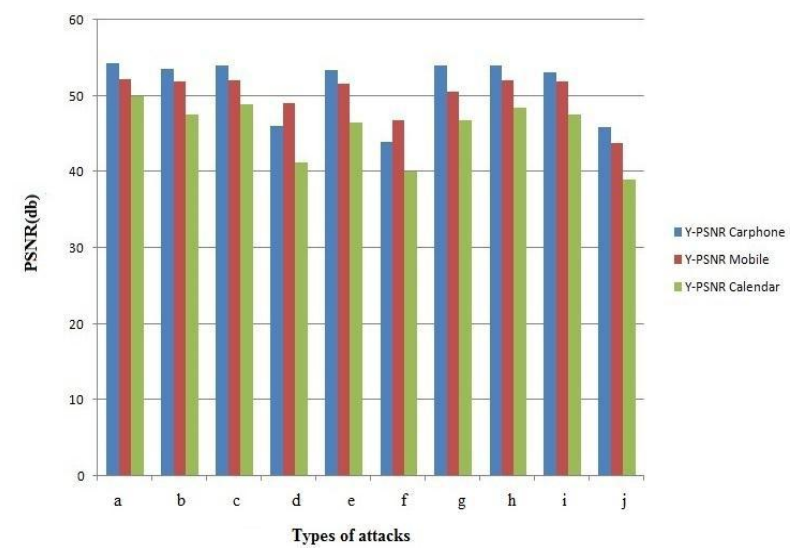

Fig 7: Disparity of Y-PSNR provoked by inserting watermark for (a)Calendar, (b)Mobile and (c)Carphone.

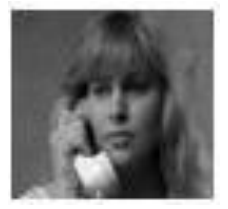

(a) Resizing

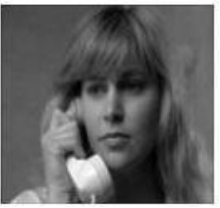

(d)Frame Averaging
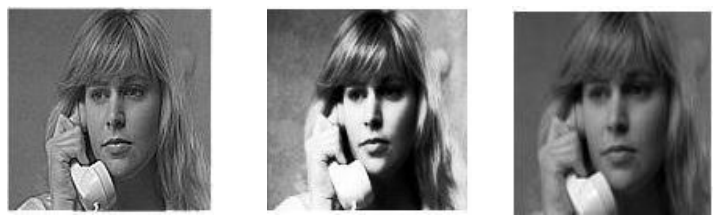

(g) Sharpening (h) Histogram Equalization (i) Frame Swapping

Fig 8: Different types of attacks applied on watermarked video
Table 1: Y-PSNR Values After Applying Different Types Of Attacks On Watermarked Video

\begin{tabular}{|l|c|c|c|}
\hline \multicolumn{1}{|c|}{ Attacks applied } & $\begin{array}{c}\text { Y-PSNR } \\
\text { Carphone }\end{array}$ & $\begin{array}{c}\text { Y-PSNR } \\
\text { Mobile }\end{array}$ & $\begin{array}{c}\text { Y-PSNR } \\
\text { Calendar }\end{array}$ \\
\hline (a) No attack & 54.23 & 52.14 & 49.98 \\
\hline (b) Resizing & 53.52 & 51.87 & 47.51 \\
\hline (c) Cropping & 45.98 & 48.98 & 41.24 \\
\hline $\begin{array}{l}\text { (d) Rotation } \\
\text { Averaging }\end{array}$ & 54.00 & 52.08 & 48.86 \\
\hline $\begin{array}{l}\text { (f) Salt \& Pepper } \\
\text { Noise }\end{array}$ & 54.02 & 51.82 & 48.52 \\
\hline $\begin{array}{l}\text { (g) Gaussian } \\
\text { Noise }\end{array}$ & 53.12 & 51.85 & 47.54 \\
\hline $\begin{array}{l}\text { (h) Sharpening } \\
\text { (i) Histogram } \\
\text { Equalization }\end{array}$ & 43.96 & 46.87 & 39.99 \\
\hline $\begin{array}{l}\text { (j) Frame } \\
\text { Swapping }\end{array}$ & 53.33 & 51.21 & 46.8 \\
\hline
\end{tabular}

\subsection{Normalized Correlation (NC)}

For comparing the similarities between the original and extracted watermarks, the two-dimensional normalized correlation (NC) value was employed. The $\mathrm{NC}$ value can be anywhere between 0 and 1 . In principle, if the $\mathrm{NC}$ value is closer to 1 , the extracted watermark is getting more similar to the embedded one. NC is computed by using Eq. (11)

$$
\frac{\sum_{i=1}^{R} \sum_{j=1}^{C}\left[V(i, j) \cdot V^{\prime}(i, j)\right]}{\sum_{i=1}^{R} \sum_{j=1}^{C}[V(i, j)]^{2}}
$$

Besides quantitative study of the robustness of the proposed approach, the visual perceptions of the extorted watermark are also required. Different type of attacks applied on watermarked videos is shown in Fig.4. As of the experimental results, it is conclude that even if the watermarked video has undergone acute physical alteration, the extorted watermark is still identifiable and the NC value is depicted in Table. 2 for unlike attacks by distinguishing with different similar methods. In this experiment, we evaluate the projected method in conflict to the related DCT-SCHUR based watermarking scheme [12] in their competence to endure disparate kinds of attacks. The eminence of the extorted watermark is resolute by NC value and the PSNR of the watermarked video. In [12], the authors apply the triangular matrix of the mark obtained after the Schur decomposition to the host image. It is not robust to all attacks and also the imperceptibility is to be improved, further it is applied to the image. 


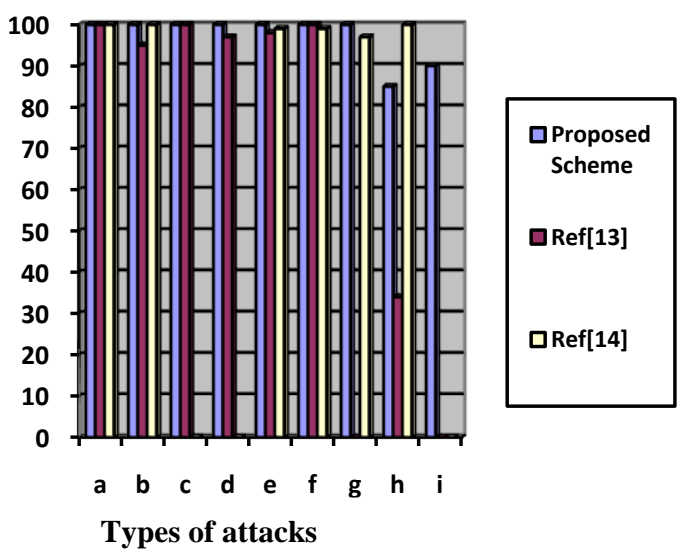

Fig 9: Comparison of NC value of proposed method with Ref[13] \& Ref[14] after sifferent types of attacks

Table 2: Relative NC Values with Earlier Methodologies.

\begin{tabular}{|l|c|c|c|}
\hline $\begin{array}{l}\text { Attacks } \\
\text { Applied }\end{array}$ & $\begin{array}{l}\text { Proposed } \\
\text { Scheme }\end{array}$ & [13] & [14] \\
\hline No attack & 1 & 1 & 1 \\
\hline Resizing & 1 & 0.98 & 1 \\
\hline Cropping & 0.95 & - & 0.8 \\
\hline Rotation & 1 & 1 & - \\
\hline $\begin{array}{l}\text { Frame } \\
\text { Averaging }\end{array}$ & 1 & 0.97 & - \\
\hline Salt \& Pepper & 1 & 0.98 & 0.99 \\
\hline $\begin{array}{l}\text { Gaussian } \\
\text { Noise }\end{array}$ & 1 & 1 & 0.99 \\
\hline Sharpening & 1 & - & 0.97 \\
\hline $\begin{array}{l}\text { Histogram } \\
\text { Equalization }\end{array}$ & 0.85 & 0.34 & 1 \\
\hline $\begin{array}{l}\text { Frame } \\
\text { Swapping }\end{array}$ & 0.90 & - & - \\
\hline
\end{tabular}

Fig.10 depicts the extracted watermark image which is similar to the original watermark image yet after dissimilar kind of attacks. Fig.11 depicts that there is no much deviation among the original videos and watermarked videos even after different types of attacks.

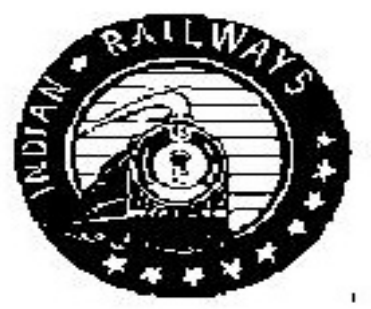

Fig 10: Extracted Watermark image after different types of attacks

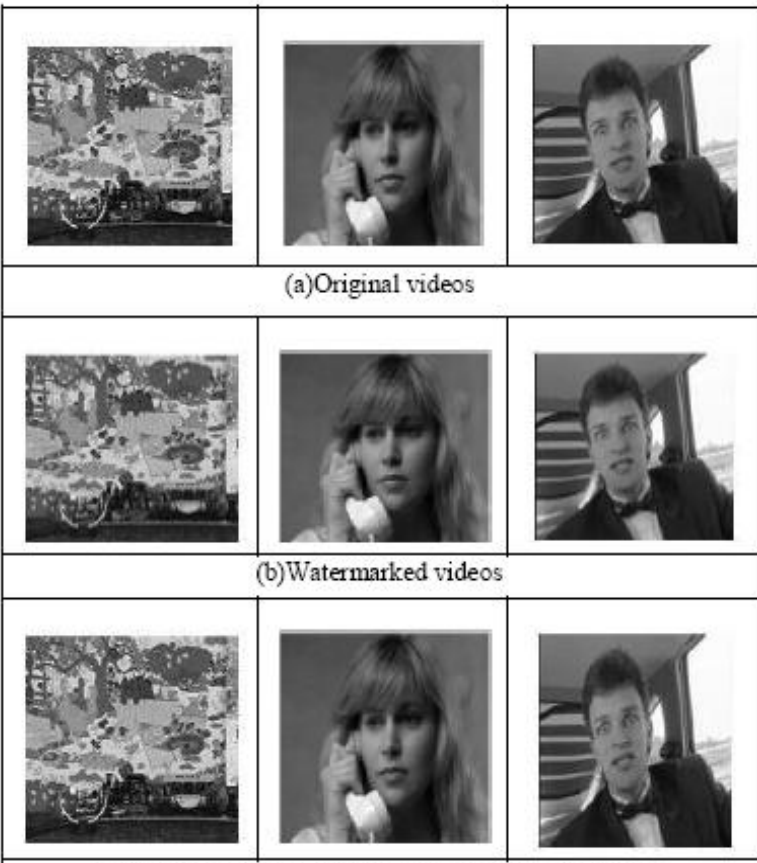

(c)Watermarked videos after different types of attacks

Figure 11 (a): Original videos, (b) Watermarked videos ,(c) Watermarked videos after different types of attacks

\section{CONCLUSION}

Fig.5. depicts the comparison of $\mathrm{NC}$ values of proposed method with Ref [13] \& Ref[14] after different types of attacks. The proposed work amalgamates the image transforms (DWT, SCHUR) with Firefly algorithm for the reason that the DWT grasp outstanding energy compaction and the watermarking by means of DWT typically reveal high-quality recital in terms of robustness and invisibility. Besides this Schur is the dominant numeric tool cooperative for applications akin to data hiding and image compression. So, the robustness to different attacks and PSNR concert of projected approach is prominent than the further related approaches. As there are very few papers to relate the results which are using this type of amalgamation on videos, so the results are compared with image watermarking techniques only. Fig.6. depicts the extracted watermark image which is similar to the original watermark image yet after dissimilar kind of attacks. Fig.11 depicts that there is no much deviation among the original videos and watermarked videos even after different types of attacks. 


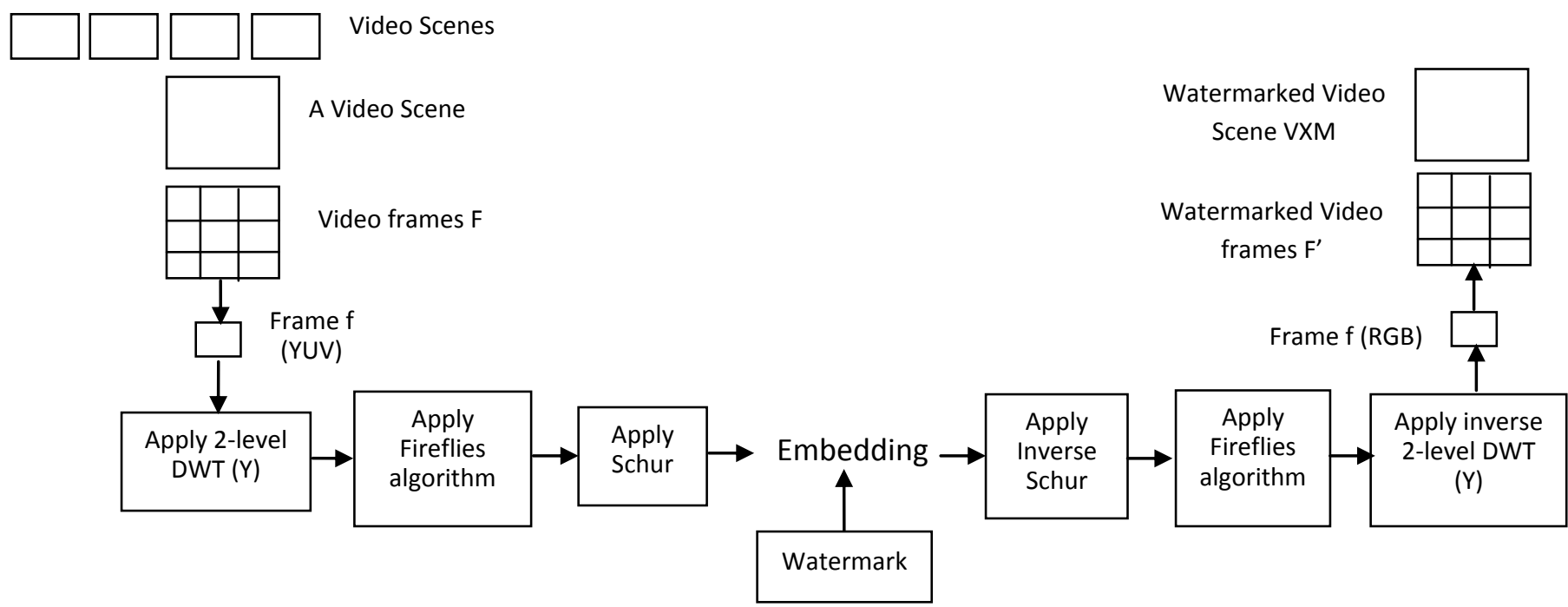

Fig 12: The watrermark embedding procedure

\section{REFERENCES}

[1] Khatib, T., Haj, A., Rajab, L. and Mohammed, H. (2008) A Robust Video Watermarking Algorithm. Journal of Compute Science, 4, 910-915.

[2] Voloshynovskiy, S., Pereira, S. and Pun, T. (2001) Attacks on Digital Watermarks: Classification Estimation-Based Attacks, and Benchmarks. Communications Magazine, 39, 118-126.

[3] Gunjal, B.L. and Manthalkar, R.R. (2010) An Overview of Transform Domain Robust Digital Image Watermarking Algorithms. Journal of Emerging Trends in Computing and Information Sciences, 2, 37-42.

[4] Xia, X.G., Boncelet, C.G. and Arce, G.R. (1997) A Multiresolution Watermark for Digital Images. Proceedings of the International Conference on Image Processing, Santa Barbara, 26-29 October 1997, 548551.

[5] Hong, W. and Hang, M. (2006) Robust Digital Watermarking Scheme for Copy Right Protection. IEEE Transactions on Signal Processing, 12, 1-8.

[6] Kundur, D. and Hatzinakos, D. (1998) Digital Watermarking Using Multiresolution Wavelet Decomposition. Proceedings of the 1998 IEEE International Conference on Acoustics, Speech and Signal Processing, Seattle, 12-15 May 1998, 2969-2972.

[7] Golub, G.H. and Van Loan, C.F. (1989) Matrix Computations. Johns Hopkins University Press, Baltimore.

[8] Mohammad, A.A. (2012) A New Digital Image Watermarking Scheme Based on Schur Decomposition. Multimedia Tools and Applications, 59, 851-883.
[9] Mohan, B.C., Swamy, K.V. and Kumar, S.S. (2011) A Comparative Performance Evaluation of SVD and Schur Decompositions for Image Watermarking. IJCA Proceedings on International Conference on VLSI, Communications and Instrumentation (ICVCI), 14, 2529.

[10] Z. A. Elizee, A. Babazadeh, S. Mohammad and S. Hosseini, "Optimizing Product Design through a Particle Swarm Induced Logistic Regression Model," Majlesi Journal of Mechanical Engineering, Vol. 3/ No. 2/ Winter-2010.

[11] E. Yang, A. T. Erdogan, T. Arslan and N. Barton, "An Improved Particle Swarm Optimization Algorithm for Power-Efficient Wireless Sensor Networks", 2007 ECSIS Symposium on bio-inspired learning and intelligent system for security, 2007.

[12] Henri Bruno Razafindradina ,Nicolas Raft Razafindrakoto, Paul Auguste Randriamitantsoa, "Improved Watermarking Scheme Using Discrete Cosine Transform and Schur Decomposition", IJCSN International Journal of Computer Science and Network, Volume 2, Issue 4, August 2013

[13] Priyanka Singh, and Suneeta Agarwal, "A Hybrid DCTSVD Based Robust watermarking scheme for copyright protection", International Conference on Emerging Trends in Engineering and Technology (ICETET'2013) Dec. 7-8, 2013 Patong Beach, Phuket (Thailand),pp.1-5.

[14] Anurag Mishra, Charu Agarwal, Arpita Sharma, Punam Bedi, "Optimized gray-scale image watermarking using DWT-SVD and Firefly Algorithm", Expert Systems with Applications 41 (2014)7858-7867. 